\title{
"Letter to the Editor: Emergency surgical treatment of total anomalous pulmonary venous connection"
}

\author{
Muhammad Sohaib ${ }^{1}$ and Arsalan Nadeem ${ }^{1}$ \\ ${ }^{1}$ Allama Iqbal Medical College
}

March 3, 2022

"Letter to the Editor: Emergency surgical treatment of total anomalous pulmonary venous connection"

Dr Muhammad Sohaib ${ }^{a}$

${ }^{a}$ Department of Medicine, Allama Iqbal Medical College, Lahore, Pakistan

Dr Arsalan Nadeem ${ }^{\mathrm{a}}$

${ }^{\mathrm{b}}$ Department of Medicine, Allama Iqbal Medical College, Lahore, Pakistan

\section{Corresponding Author:}

Dr Muhammad Sohaib Address: Department of Medicine, Allama Iqbal Medical College, Allama Shabbir Ahmad Usmani Road, Lahore, Punjab, Pakistan 54550 Tel: +92 3208047533 Email: sohaibrehmani@hotmail.com

\section{Co-authors:}

Dr Arsalan Nadeem Address: Department of Medicine, Allama Iqbal Medical College, Allama Shabbir Ahmad Usmani Road, Lahore, Punjab, Pakistan 54550 Tel: +92 3452780767 Email: arsalannadeemh@gmail.com

Article category: Letter to the editor

Manuscript word count: 424 words

Number of references: 4

Number of tables: 0

Number of figures: 0

Keywords:complete anomalous pulmonary venous connection; congenital heart disease; emergency operation; long-term follow-up; pulmonary venous obstruction

\section{Letter:}

To the Editor,

We meticulously read your article titled "Emergency surgical treatment of total anomalous pulmonary venous connection" by Yongtao Wu MD et $\mathrm{al}^{1}$ and we sincerely congratulate the authors for their appreciable efforts.

As substantiated by diverse research on the impact of emergency surgery in patients with total anomalous pulmonary venous connection (TAPVC), ${ }^{2}$ we agree with the conclusion of the study that emergency surgery achieves beneficial short term results in TAPVC patients. However, we consider it essential to mention 
additional noteworthy points that would enhance the quality of this article and add to existing knowledge of this crucial surgical procedure.

Firstly, we observed that key baseline characteristics and variables were missing from the study. A recent retrospective study analyzing the impact of emergency surgery without stabilization on TAPVC included gestational age and preoperative indicators such as preoperative lowest $\mathrm{pH}$, highest plasma lactate, aspartate aminotransferase, peak creatinine, and peak urea. ${ }^{2}$ Additionally, in another study, ${ }^{3}$ patients were characterized on the basis of their presentation in the emergency ward, their ventilation status and prior administration of inotropic agents. We believe including these characteristics would have further allowed the authors of the original study to better stratify patients and predict the impact of emergency surgery on them.

Secondly, the authors mentioned several common post-operative complications of this emergency procedure, yet missed some complications such as hospital acquired infections, low cardiac output \& diaphragmatic paralysis. ${ }^{2}$ An assessment of these complications would have further increased the validity of the original study.

Thirdly, a 2021 retrospective study indicated sutureless technology as an alternate treatment option for TAPVC. Sutureless technology improved surgical outcomes for patients with infracardiac TAPVC, preoperative pulmonary venous obstruction, low body weight, or complicated anatomy. ${ }^{4}$ These observations are consistent with previous studies establishing that sutureless technology is more suitable for high-risk patients with obstructive, infracardiac or mixed TAPVC. ${ }^{4}$ The authors should have explored promising effects of such technology making it a potential therapeutic application for TAPVC.

Fourthly, the authors failed to assess central venous pressure (CVP) which is a crucial risk factor for postoperative death in TAPVC patients undergoing surgical treatment. ${ }^{4}$ In a previous study, the patients were divided into three equal cohorts according to postoperative CVP and it was found that when postoperative CVP was greater than $8 \mathrm{mmHg}$, early and overall mortality increased significantly. ${ }^{4}$ This is consistent with findings observed in previous studies. ${ }^{4}$ Finally, polycentric approaches should be adopted to enhance early diagnosis and appropriate treatment as well as ingenious therapies explored to yield alternate treatment options.

\section{References:}

1. Wu Y, Fan X, Chen L, Wang D, Su J, Jin C, Wang Z. Emergency surgical treatment of total anomalous pulmonary venous connection. J Card Surg. 2022 Jan;37(1):47-52. doi: 10.1111/jocs.16079. Epub 2021 Oct 20. PMID: 34669211.

2. Xi L, Wu C, Pan Z, Xiang M. Emergency surgery without stabilization prior to surgical repair for total anomalous pulmonary venous connection reduces duration of mechanical ventilation without reducing survival. J Cardiothorac Surg. 2021 Aug 2;16(1):213. doi: 10.1186/s13019-021-01559-y. PMID: 34340695; PMCID: PMC8328139.

3. Harada T, Nakano T, Oda S, Kado H. Surgical results of total anomalous pulmonary venous connection repair in 256 patients. Interact Cardiovasc Thorac Surg. 2019 Mar 1;28(3):421-426. doi: 10.1093/icvts/ivy267. PMID: 30202975.

4. Ji E, Qiu H, Liu X, Xie W, Liufu R, Liu T, Chen J, Wen S, Li X, Cen J, Zhuang J. The Outcomes of Total Anomalous Pulmonary Venous Connection in Neonates-10-Year Experience at a Single Center. Front Cardiovasc Med. 2021 Nov 12;8:775578. doi: 10.3389/fcvm.2021.775578. PMID: 34869690; PMCID: PMC8632761.

\section{Acknowledgements:}

None to declare.

CRediT author statement (author contribution): 
Dr Muhammad Sohaib: conceptualization, data curation, methodology, software, writing - original draft preparation.

Dr Arsalan Nadeem: supervision, software, writing - reviewing and editing.

Disclaimer:

None to declare.

Declarations of interest:

None.

Funding statement:

This research did not receive any specific grant from funding agencies in the public, commercial, or not-forprofit sectors. 